\title{
Application of a two-fluid model to simulate the heating of two-phase flows.
}

Jean-Marc Hérard and Olivier Hurisse and Antoine Morente and Khaled Saleh

\begin{abstract}
This paper is dedicated to the simulation of two-phase flows on the basis of a two-fluid model that allows to account for the disequilibrium of velocities, pressures, temperatures and chemical potentials (mass transfer). The numerical simulations are performed using a fractional step method treating separately the convective part of the model and the source terms. The scheme dealing with the convective part of the model follows a Finite Volume approach and is based on a relaxation scheme. In the sequel, a special focus is put on the discretization of the terms that rule the mass transfer. The scheme proposed is a first order implicit scheme and can be verified using an analytical solution. Eventually, a test case of the heating of a mixture of steam and water is presented, which is representative of a steam generator device.
\end{abstract}

\section{Introduction}

Most of the industrial processes used for generating electricity require the use of fluids, and especially water. The water is used either as a coolant fluid or to ensure the production of mechanical work through the turbines which are motionned by steam. If we focus on a nuclear power plant based on a Pressurized Water Reactor (PWR), the water is used as liquid or vapour depending on the circuit under consideration. In particular, the secondary circuit of a nuclear power plant contains steam

Hérard Jean-Marc

EDF R\&D, 6 Quai Watier, 78400 Chatou, France, e-mail: jean-marc.herard@edf.fr

Hurisse Olivier

EDF R\&D, 6 Quai Watier, 78400 Chatou, France, e-mail: olivier.hurisse@edf.fr

Morente Antoine

EDF R\&D, 6 Quai Watier, 78400 Chatou, France, e-mail: antoine.morente@edf.fr

Saleh Khaled

IRSN, BP-3 13115 Saint-Paul-lez-Durance cedex, France, e-mail: khaled.saleh@irsn.fr 
and liquid water. Moreover, vaporization and condensation phenomena take place in different parts of that circuit. In this industrial context, the two fluid approach is often retained to perform fine 3D simulations in complex geometries.

For instance, the well-known standard two-fluid model [9] is widely used in industrial numerical codes. This model allows to deal with the velocity and temperature disequilibrium, and to take into account the mass transfer between the phases by a source term measuring the distance to the saturation (most of the time in terms of enthalpy or temperaure). In this model, the pressure is assumed to be the same for the two phases at every point and every time. This pressure equilibrium is based on the mechanical assumption of large interfaces between the two phases [9] and it neglects the thermodynamical aspect of the pressure equilibrium. Indeed, the classical thermodynamics theory states that two phases of the same fluid are in equilibrium if and only if: the pressures, the temperatures and the chemical potentials are equal for the two phases. In our opinion, it is crucial to recover this equilibrium condition in a model used to perform numerical simulations of two-phase flows, mainly if mass transfer is an important feature of the problem. We thus choose a model that also takes explicitely into account the pressure disequilibrium between the phases.

The two-fluid model used in the sequel is related to the so-called Baer-Nunziato model $[1,10]$. Its formal derivation has been performed following a statistical approach in [8]. In one space dimension, the corresponding system possesses seven independent variables: the statistical fraction of liquid, the statistical mean temperatures, the statistical mean pressures and the statistical mean velocities. The spacetime evolution of these variables is described by a set a PDEs whose convective part is hyperbolic and whose source terms are chosen to comply with the entropy inequality, based on the physical mixture entropy. Non-conservative products are present in the equations but some specific closures [4] allow to define discontinuous solutions in a unique manner.

The whole numerical scheme proposed here is based on a operator-splitting method [15]. We first account for the convective part of the system thanks to the explicit relaxation scheme proposed in $[2,13]$. The source terms are then successively discretized by four implicit ODE schemes. Very good agreement with experiments has been found in [11] (using a Rusanov scheme for the convective part) focusing on situations where the mass transfer occurs due to a pressure drop. We propose here a one-dimensional test case close to the OECD test case [14]: the mass transfer is due to the heating of saturated water which flows in a pipe.

\section{The two-fluid model}

The system of PDEs governing the time-space evolution of the variables is: 


$$
\left\{\begin{array}{l}
\partial_{t}\left(\alpha_{v}\right)+V_{i}(W) \partial_{x}\left(\alpha_{v}\right)=\phi_{v}(W), \\
\partial_{t}\left(m_{l}\right)+\partial_{x}\left(m_{l} U_{l}\right)=-\Gamma_{v}(W), \\
\partial_{t}\left(m_{l} U_{l}\right)+\partial_{x}\left(m_{l} U_{l}^{2}+\alpha_{l} P_{l}\right)-P_{i}(W) \partial_{x}\left(\alpha_{l}\right)=-D_{v}(W)-\Gamma_{v}(W) \bar{U}_{i n t}, \\
\partial_{t}\left(\alpha_{l} E_{l}\right)+\partial_{x}\left(\alpha_{l} U_{l}\left(E_{l}+P_{l}\right)\right)+P_{i}(W) \partial_{t}\left(\alpha_{l}\right)=-\psi_{v}(W)-\bar{V}_{i n t} D_{v}(W)-\Gamma_{v}(W) \bar{H}_{i n t}, \\
\partial_{t}\left(m_{v}\right)+\partial_{x}\left(m_{v} U_{v}\right)=\Gamma_{v}(W) \\
\partial_{t}\left(m_{v} U_{v}\right)+\partial_{x}\left(m_{v} U_{v}^{2}+\alpha_{v} P_{v}\right)-P_{i}(W) \partial_{x}\left(\alpha_{v}\right)=D_{v}(W)+\Gamma_{v}(W) \bar{U}_{i n t}, \\
\partial_{t}\left(\alpha_{v} E_{v}\right)+\partial_{x}\left(\alpha_{v} U_{v}\left(E_{v}+P_{v}\right)\right)+P_{i}(W) \partial_{t}\left(\alpha_{v}\right)=\psi_{v}(W)+\bar{V}_{i n t} D_{v}(W)+\Gamma_{v}(W) \bar{H}_{i n t},
\end{array}\right.
$$

where $\alpha_{k}$ denote the statistical fractions and satisfy $\alpha_{l}+\alpha_{v}=1, \rho_{k}$ denote the densities, $m_{k}=\alpha_{k} \rho_{k}$ are the partial masses, $U_{k}$ the velocities, $P_{k}$ the pressures and $E_{k}$ the total energies which read $E_{k}=\rho_{k}\left(e_{k}+U_{k}^{2} / 2\right)$. The specific internal energies $e_{k}$ are obtained through an EOS defined with respect to the pressures and densities: $e_{k}=e_{k}\left(\rho_{k}, P_{k}\right)$. Closure laws have to be provided for the velocities $V_{i}(W), \bar{V}_{\text {int }}(W), \bar{U}_{\text {int }}(W)$, for the pressure $P_{i}(W)$ and for the energy $\bar{H}_{\text {int }}(W)$, where $W=\left(\alpha_{l}, m_{l}, m_{l} U_{l}, \alpha_{l} E_{l}, m_{v}, m_{v} U_{v}, \alpha_{v} E_{v}\right)$. We follow the choice proposed in [4, 6, 7]: $V_{i}(W)=U_{v}, P_{i}(W)=P_{l}, \bar{U}_{i n t}=\bar{V}_{\text {int }}=\left(U_{l}+U_{v}\right) / 2$ and $\bar{H}_{\text {int }}=U_{l} U_{v} / 2$. We also define the total mass $m=m_{l}+m_{v}$, the mean velocity $U$ with $m U=m_{l} U_{l}+m_{v} U_{v}$, and the total energy of the mixture $E=\alpha_{l} E_{l}+\alpha_{v} E_{v}$.

The source terms for the pressure relaxation $\phi_{v}(W)$, for the mass transfer $\Gamma_{v}(W)$, for the drag force $D_{v}(W)$ and for the heat exchange $\psi_{v}(W)$ are then chosen according to the entropy inequality for the mixture $s=m_{l} s_{l}\left(\rho_{l}, P_{l}\right)+m_{v} s_{v}\left(\rho_{v}, P_{v}\right)$ and the associated entropy-flux $\eta_{s}=m_{l} U_{l} s_{l}\left(\rho_{l}, P_{l}\right)+m_{v} U_{v} s_{v}\left(\rho_{v}, P_{v}\right)$, where $s_{k}$ are the physical phasic specific entropies. The source terms can then be chosen as:

$$
\begin{aligned}
& \Gamma_{v}(W)=\frac{1}{\tau_{g}(W)} \frac{m_{l} m_{v}}{\left(m_{l}+m_{v}\right)\left(\left|\mu_{v}\right| / T_{v}+\left|\mu_{l}\right| / T_{l}\right)}\left(\mu_{l} / T_{l}-\mu_{v} / T_{v}\right), \\
& D_{v}(W)=\frac{1}{\tau_{u}(W)} \frac{m_{l} m_{v}}{m_{l}+m_{v}}\left(U_{l}-U_{v}\right), \\
& \psi_{v}(W)=\frac{1}{\tau_{t}(W)} \frac{m_{l} C_{V, l} m_{v} C_{V, v}}{m_{l} C_{V, l} l m_{v} C_{V, v}}\left(T_{l}-T_{v}\right), \\
& \phi_{v}(W)=\frac{\alpha_{l} \alpha_{v}}{K_{p}(W)}\left(P_{v}-P_{l}\right),
\end{aligned}
$$

with the positive characteristic time scales $\tau_{g}, \tau_{u}, \tau_{t}$, and the positive parameter $K_{p}$ which has the dimension of a kinematic vicosity [5]. The chemical potentials are denoted by $\mu_{k}=e_{k}+P_{k} / \rho_{k}-T_{k} s_{k}, T_{k}=T_{k}\left(\rho_{k}, P_{k}\right)$ stand for the temperatures and $C_{V, k}$ are the specific heat capacities.

Model (1) with the closures proposed above is defined for a statistical liquid fraction in ]0,1[. Otherwise, if for instance $\alpha_{l}=0$, the quantities $\rho_{l}, U_{l}$ and $e_{l}$ are not defined in a unique manner. It is important to note that due to the choice of the closures for (1), $\alpha_{l}$ remains in ]0,1[ if the initial condition for $\alpha_{l}$ belongs to ]0,1[ everywhere on the spacial domain and if $\alpha_{l}$ is in ] 0,1 [ on the boundary of the domain (especially at the inlets). Other properties of this model can be found in $[3,4,8]$. 
With this model, the thermodynamical equilibrium is reached if and only if the temperatures, the pressures and the chemical potentials are equal. In the pressuretemperature plane, the set of couples $(P, T)$ which are solutions of the system:

$$
T_{l}=T=T_{v}, \quad P_{l}=P=P_{v}, \quad \frac{\mu_{l}\left(T_{l}, P_{l}\right)}{T_{l}}=\frac{\mu_{v}\left(T_{v}, P_{v}\right)}{T_{v}} \Leftrightarrow \mu_{l}(T, P)=\mu_{v}(T, P),
$$

represents the so-called saturation curves for which the two phases co-exist in a stable manner. For any couple $(P, T)$ which is not solution of (3), only one of the two phases is stable (i.e. the other one tends to vanish). When considering Stiffened Gas EOS in the pressure-temperature plane, the chemical potential reads:

$$
\begin{aligned}
& \frac{\mu_{k}\left(T_{k}, P_{k}\right)}{T_{k}}=\gamma_{k} C_{V, k}-s_{k}\left(T_{k}, P_{k}\right), \\
& s_{k}\left(T_{k}, P_{k}\right)=s_{k, 0}+\gamma_{k} C_{V, k} \ln \left(C_{V, k} T_{k}\right)-\left(\gamma_{k}-1\right) C_{V, k} \ln \left(\frac{P_{k}+P_{i n f, k}}{\gamma_{k}-1}\right),
\end{aligned}
$$

where $\gamma_{k}>1, C_{V, k}$ and $P_{\text {inf }, k}$ are constant. We can exhibit explicitely the saturation curve for the temperature with respect to the pressure. It is defined only if $\gamma_{v} C_{V, v} \neq$ $\gamma_{l} C_{V, l}$ and reads:

$$
T_{\text {sat }}(P)=e^{\left(\frac{\beta_{l}-\beta_{v}+\gamma_{v} C_{V, v}-\gamma_{l} C_{V, l}}{\gamma_{v} C_{V, v}-\gamma_{l} C_{V, l}}\right)}\left(\frac{\left(P+P_{i n f, v}\right)^{\left(C_{V, v}\left(\gamma_{v}-1\right)\right)}}{\left(P+P_{i n f, l}\right)^{\left(C_{V, l}\left(\gamma_{l}-1\right)\right)}}\right)^{\left(\frac{1}{\gamma_{v} C_{V, v}-\gamma_{l} C_{V, l}}\right)},
$$

where $\beta_{k}=s_{k, 0}+\gamma_{k} C_{V, k} \ln \left(C_{V, k}\right)+\left(\gamma_{k}-1\right) C_{V, k} \ln \left(\gamma_{k}-1\right)$ are the constant parts of the entropies $s_{k}\left(P_{k}, T_{k}\right)$. The saturation curve for the pressure with respect to the temperature can not be written explicitely.

\section{Discretization scheme}

The overall scheme is based on a fractionnal step method [15]. We first account for the convection terms, which corresponds to system (1) with $\Gamma_{v}(W)=D_{v}(W)=$ $\psi_{v}(W)=\phi_{v}(W)=0$. In the sequel, this step is achieved using the relaxation scheme described in [2]. It is not recalled here and the convergence curves obtained for analytical test cases can be found in [12]. This scheme has proven to be accurate and has shown good capability to treat small values of $\alpha_{k}$, which are very important features for industrial simulations.

In the second step of the algorithm, source terms $\Gamma_{v}(W), D_{v}(W), \psi_{v}(W)$ and $\phi_{v}(W)$ are accounted for successively through the corresponding ODE system with the time step $\Delta t$ fixed by the convection scheme. The corresponding schemes for $D_{v}(W), \psi_{v}(W)$ and $\phi_{v}(W)$ are implicit and are described in [7, 11, 12]. For each source term, analytical solutions can also be found in these references. We focus here on the scheme that handles the mass transfer term $\Gamma_{v}(W)$. Paying attention to the properties of mass, momentum and total energy conservation for the mixture, 
the ODE system for the mass transfer obtained from system (1) is:

$$
\left\{\begin{array}{l}
\partial_{t}\left(\alpha_{v} \rho_{v}\right)=\Gamma_{v} \\
\partial_{t}\left(\alpha_{v} \rho_{v} U_{v}\right)=\bar{U}_{i n t} \Gamma_{v} \\
\partial_{t}\left(\alpha_{v} E_{v}\right)=\bar{H}_{i n t} \Gamma_{v} \\
\partial_{t} \alpha_{v}=\partial_{t}(m)=\partial_{t}(m U)=\partial_{t}(E)=0
\end{array}\right.
$$

Starting from an initial value $W^{n}$ of $W$ at time $t^{n}$, we describe now how the value $W^{n+1}$ is computed at time $t^{n+1}=t^{n}+\Delta t$.

We first approximate system (6) by taking $\tau_{g}=\tau_{g}(W(t=0))$. The solutions for the statistical fractions are obvious: $\alpha_{k}(t)=\alpha_{k}(t=0)$, which enables to write the source term $\Gamma_{v}$ as a function of the densities and the specific internal energies: $\Gamma_{v}=\tilde{\Gamma}_{v}\left(\rho_{l}, e_{l}, \rho_{v}, e_{v}\right)$. Moreover, thanks to the closures for $\bar{U}_{\text {int }}$ and $\bar{H}_{\text {int }}$, the internal energies remain constant:

$$
\partial_{t}\left(m_{v} e_{v}\right)=0 \quad \text { and } \quad \partial_{t}\left(m_{l} e_{l}\right)=0
$$

If we now use the fact that the mass of the mixture is conserved, $\Gamma_{v}$ can be written as a function of $m_{l}(t)$ (or $\left.m_{v}(t)\right)$ and the initial conditions:

$$
\begin{aligned}
\Gamma_{v} & =\tilde{\Gamma}_{v}\left(\rho_{l}, e_{l}, \rho_{v}, e_{v}\right) \\
& =\tilde{\Gamma}_{v}\left(\frac{m_{l}}{\alpha_{l}(t=0)}, \frac{\left(m_{l} e_{l}\right)(t=0)}{m_{l}}, \frac{\left(m_{l}+m_{v}\right)(t=0)-m_{l}}{\alpha_{v}(t=0)}, \frac{\left(m_{v} e_{v}\right)(t=0)}{\left(m_{l}+m_{v}\right)(t=0)-m_{l}}\right) \\
& =\bar{\Gamma}_{v}\left(m_{l}\right) .
\end{aligned}
$$

A straightforward consequence of this property is that the mass equation (i.e. the first equation of (6)) can be solved independently of the other equations. In general, the source term $\Gamma_{v}$ can not be explicitely integrated. We thus solve the mass equation using one time-step $\Delta t$ of the Euler implicit scheme:

$$
m_{l}^{n+1}=m_{l}^{n}-\Delta t \bar{\Gamma}_{v}\left(m_{l}^{n+1}\right), \quad \text { with } \quad m_{l}^{n+1} \in\left[0, m_{l}^{n}+m_{v}^{n}\right] .
$$

The solution $m_{l}^{n+1}$ at the end of the time step may be computed by a dichotomy algorithm. The function $Y \rightarrow \bar{\Gamma}_{v}(Y)$ is non-linear and might be non smooth. We can state the following result setting $F(Y)=m_{l}^{n}-Y-\Delta t \bar{\Gamma}_{v}(Y)$. Since $\Gamma_{v}$ vanishes for $m_{l}=0$ or $m_{v}=0$ we obviously have $F(0)=m_{l}^{n}$ and $F\left(m_{l}^{n}+m_{v}^{n}\right)=-m_{v}^{n}$. If we assume that $Y \rightarrow \bar{\Gamma}_{v}(Y)$ is continuous, its form ensures that if the masses $m_{k}^{n}$ are positive, then the masses $m_{k}^{n+1}$ are also positive. Finally, if $F$ is continuous and strictly monotone on $\left[0, m_{l}^{n}+m_{v}^{n}\right]$, there exists a unique solution to (9) in $] 0, m_{l}^{n}+$ $m_{v}^{n}$. Once the mass $m_{l}^{n+1}$ has been computed, the term $\bar{\Gamma}_{v}\left(m_{l}^{n+1}\right)$ and the remaining equations can be updated using one step of the implicit Euler scheme: 


$$
\left\{\begin{array}{l}
\alpha_{l}^{n+1}=\alpha_{l}^{n}, \quad m_{l}^{n+1}=m_{l}^{n}-\Delta t \bar{\Gamma}_{v}\left(m_{l}^{n+1}\right), \quad m_{v}^{n+1}=m^{n}-m_{l}^{n+1}, \\
\left(m_{l} U_{l}\right)^{n+1}=\left(m_{l} U_{l}\right)^{n}-\Delta t \bar{U}_{i n t}^{n+1} \bar{\Gamma}_{v}\left(m_{l}^{n+1}\right), \quad\left(m_{v} U_{v}\right)^{n+1}=m U_{l}^{n}-\left(m_{l} U_{l}\right)^{n+1}, \\
\left(\alpha_{l} E_{l}\right)^{n+1}=\left(\alpha_{l} E_{l}\right)^{n}-\Delta t \bar{H}_{i n t}^{n+1} \bar{\Gamma}_{v}\left(m_{l}^{n+1}\right), \\
\left(\alpha_{v} E_{v}\right)^{n+1}=\left(\alpha_{v} E_{v}\right)^{n}+\left(\alpha_{l} E_{l}\right)^{n}-\left(\alpha_{l} E_{l}\right)^{n+1} .
\end{array}\right.
$$

In fact, the two momentum equations form a $2 \times 2$ linear system whose determinant $\Delta_{G, u}$ is always positive if and only if the partial masses are positive, since: $\Delta_{G, u}=\left(m_{l}^{n} m_{v}^{n+1}+m_{l}^{n+1} m_{v}^{n}\right) / 2$. Once the velocities $U_{k}^{n+1}$ are known, the update of the total energies $E_{l}^{n+1}$ and $E_{v}^{n+1}$ is straightforward. This scheme is a first-order scheme which ensures the conservation of the total mass, the total momentum and the total energy of the mixture. The positivity of the fractions and the partial masses is ensured.

\section{Heated saturated water in a pipe}

The test case is derivated from the OECD/CSNI benchmark problem [14]. It consists in heating saturated water flowing in a one-dimensional pipe. The increase of heat of the fluid leads to vaporization of the water which is advected. The sketch of the case is depicted in figure (1). Since we do not account for the head loss in the pipe - as proposed in the OECD/CSNI benchmark problem - we do not need to wait for a stationnary state to be established in the pipe before beginning to heat the fluid. In fact the initial conditions given below already represent a stationnary state. Hence the time schedule of the present case is slightly different.

The initial conditions are chosen at a pressure of $P=71.0$ bars and a temperature close to the saturation temperature $T=559.75 \mathrm{~K}$. They are: $\alpha_{l}=0.99$, $\rho_{l}=739.8 \mathrm{~kg} / \mathrm{m}^{3}, \rho_{v}=37.1 \mathrm{~kg} / \mathrm{m}^{3}, U_{l}=U_{v}=1.468 \mathrm{~m} / \mathrm{s}, P_{l}=P_{v}=71.0$ bars. The EOS parameters are chosen to get these values and to recover the values of the phasic celerities and a temperature saturation-curve (5) close to the real one in the vicinity of the pressure $P=71.0$ bars and the temperature $T=559.75 \mathrm{~K}$. It yields: $C_{V, v}=1329.45 \mathrm{~J} / \mathrm{kg} / \mathrm{K}, \gamma_{v}=1.257, P_{\text {inf }, v}=0, s_{v}^{0}=-16274.14 \mathrm{~J} / \mathrm{kg} / \mathrm{K}$, $C_{V, l}=285.14 \mathrm{~J} / \mathrm{kg} / \mathrm{K}, \gamma_{l}=3, P_{\text {inf }, l}=2.2910^{8}$ bars, $s_{l}^{0}=0$. The saturation curve is shown on figure (2) together with a tabulated saturation curve. The difference is not negligible. Actually, due to the higher slope of the stiffened gas saturation curve, we may underestimate the vapour production. For the inlet boundary-condition the values are the same as the initial condition values. These values provide an equilibrium state since velocities, pressures, temperatures and chemical potentials are equal.

We are interested in the stationnary state that is reached after 10 seconds of physical time. The results obtained with the code presented in the previous sections are given on figures (3) and (4). They correspond to an industrial mesh with 200 uniform cells. The CFL condition $1 / 2$ applied to the convection scheme leads to a time step of $1.510^{-5} \mathrm{~s}$. The latter is smaller than the time scales which are: $\tau_{g}=2.010^{-4} \mathrm{~s}$ 
and $\tau_{t}=1.010^{-4} \mathrm{~s}$. The parameter for pressure relaxation is chosen in accordance with [5]: $K_{p}=1.22610^{-4}$. Figure (3) represents the thermodynamical variables along the $\mathrm{x}$-axis at time $t=10 \mathrm{~s}$ and Figure (4) gives the vapour mass fraction and the mixture mass flow rate at the outlet of the domain for the whole simulation time. It can be noticed that the heating mainly results in the increase of the temperature and that the pressures do not vary a lot. At the outlet of the domain, the liquid fraction starts to evolve at time $t=1 \mathrm{~s}$, which corresponds to the time necessary for the vapour generated to reach the outlet (the vapour travels at almost $1.4 \mathrm{~m} / \mathrm{s}$ and there is almost $1.4 \mathrm{~m}$ between the downstream edge of the heating zone and the outlet).

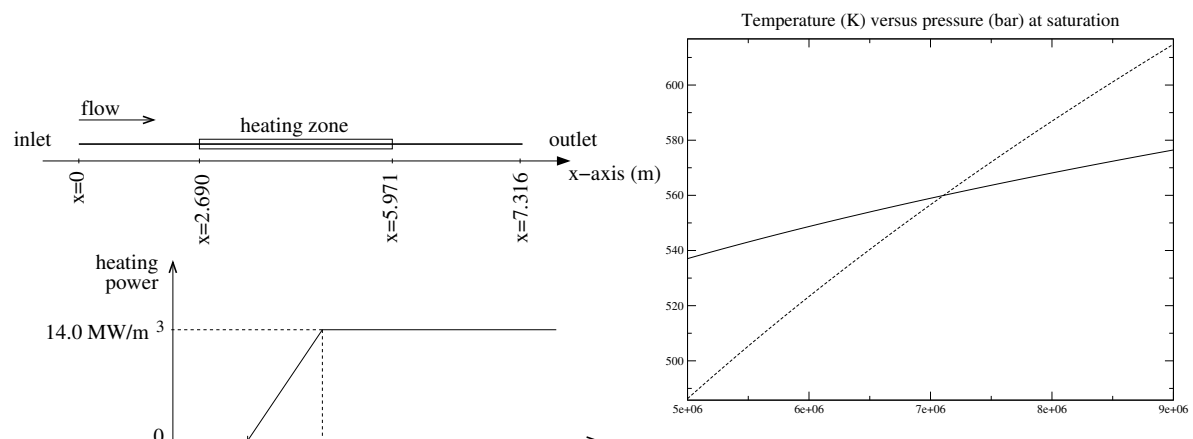

Fig. 1 Sketch of the test case: geometrical domain and time-schedule of the heating.

Fig. 2 Saturation curves for the temperature with respect to the pressure on the interval [50 bars, 90 bars]. The plain line represents a reference saturation curve, whereas the dashed line represents the saturation curve obtained with our stiffened gas EOS.
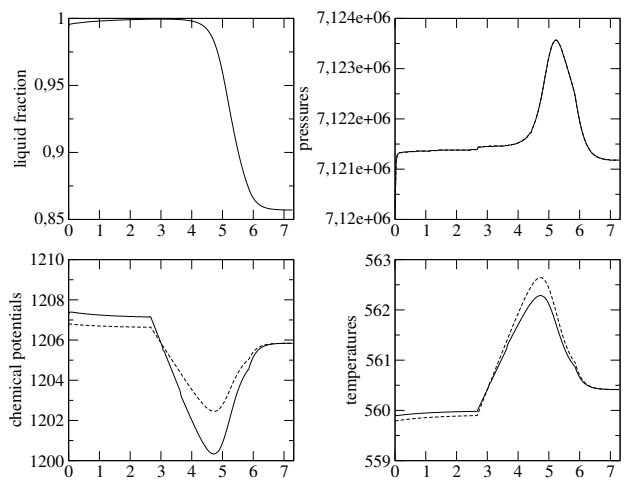

Fig. 3 Thermodynamical variables along the x-axis at time $t=10 \mathrm{~s}$. The plain lines represent the liquid variables and the dashed lines represent the vapour variables. 

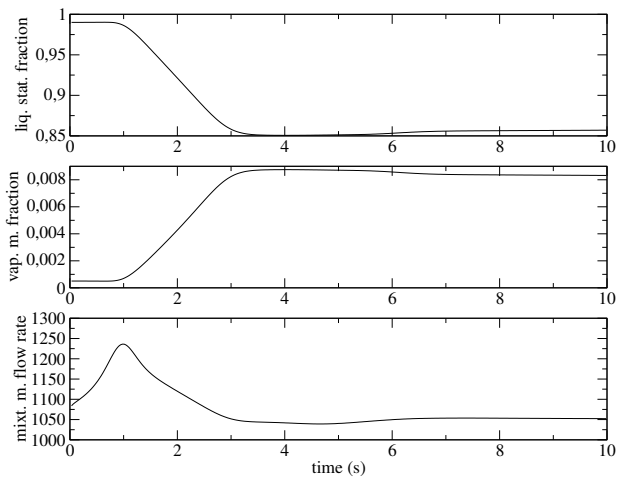

Fig. 4 Liquid statistical fraction, vapour mass fraction and mixture mass flow rate at the outlet of the domain along the simulation.

\section{References}

1. Baer, M., Nunziato, J.W.: A two-phase mixture theory for the deflagration-to-detonation transition in reactive granular materials. International Journal of Multiphase Flows 12, 861-889 (1986)

2. Coquel, F., Hérard, J.M., Saleh, K., Seguin, N.: A robust entropy-satisfying finite volume scheme for the isentropic Baer Nunziato model. Mathematical Modelling and Numerical Analysis 48(1), 165-206 (2014)

3. Coquel, F., Hérard, J.M., Saleh, K., Seguin, N.: Two properties of two-velocity two-pressure models for two-phase flows. Comm. in Math. Scien. 12(3), 593-600 (2014)

4. Gallouët, T., Hérard, J.M., Seguin, N.: Numerical modeling of two-phase flows using the twofluid two-pressure approach. Mathematical Models and Methods in Applied Sciences 14(5), 663-700 (2004)

5. Gavrilyuk, S.: The structure of pressure relaxation terms: one velocity case. EDF R\&D report H-I83-2014-00276-EN

6. Hérard, J.M., Hurisse, O.: Computing two-fluid models of compressible water-vapour flows with mass transfer. http://www.aiaa.org, AIAA paper 2012-2959 (2012)

7. Hérard, J.M., Hurisse, O.: A fractional step method to compute a class of compressible gasliquid flows. Computers and Fluids 55, 57-69 (2012)

8. Hérard, J.M., Liu, Y.: Une approche bifluide statistique de modélisation des écoulements diphasiques à phases compressibles. EDF R\&D report H-I81-2013-01162-FR (2013)

9. Ishii, M., Hibiki, T.: Thermo-fluid dynamics of two-phase flow. Springer (2006)

10. Kapila, A., Bdzil, J., Menikoff, R., Son, S., Stewart, D.: Two-phase modelling of ddt in granular materials : reduced equations. Physic of Fluids 13, 3002-3024 (2001)

11. Liu, Y.: Contribution à la vérification et à la validation d'un modèle diphasique bifluide instationnaire. PhD thesis, Aix Marseille University, http://tel.archives-ouvertes.fr/tel-00864567 $(11 / 09 / 2013)$

12. Morente, A., Hurisse, O., Hérard, J.M.: Vérification d'un code pour les écoulements diphasiques. EDF R\&D report H-I83-2013-03283-FR (2013)

13. Saleh, K.: Analyse et simulation par relaxation d'écoulements diphasiques compressibles. $\mathrm{PhD}$ thesis, Pierre et Marie Curie University, http://tel.archives-ouvertes.fr/tel-00761099 $(26 / 11 / 2012)$

14. Werner, W.: First CSNI numerical benchmark problem. Comparison report. CSNI report 47 (1980)

15. Yanenko, N.N.: Méthodes à pas fractionnaires. Armand Colin (1968) 